


\section{Las elecciones olvidadas: \\ Reflexiones en torno a la \\ Participación ciudadana \\ En elecciones de diputados \\ Locales en el estado \\ De hidalgo (1993-2005)}

Enrique López Rivera *

\section{Resumen}

Las elecciones de diputados locales son las que concentran el mayor índice de abstencionismo en el estado de Hidalgo. Ese comportamiento puede ser explicado a través de algunos factores de corte sociodemográfico que ayudan a comprender los contrastes y contradicciones de la conducta del votante y del abstencionista. ¿Cuáles de factores ayudan a entender con mayor precisión este fenómeno? Esa es la pregunta que trata de contestar el presente texto.

\section{Abstract}

The elections of local deputies are those that concentrate the major index of abstentionism in the state of Hidalgo. This behavior can be made clear across some factors of court sociodemográfico that they help to understand the contrasts and contradictions of the conduct of the voter and of the abstentionist. Which of factors do help to understand with major precision this phenomenon? This it is the question that tries to answer the present text.

\section{Introducción}

Tradicionalmente en el estado de Hidalgo las elecciones locales tienen menor asistencia que las elecciones federales. Entre ellas, la renovación del Congreso Local es la que mayor abstencionismo concentra. Los ciudadanos prefieren participar más en votaciones federales (principalmente en las presidenciales) porque son más competidas o bien, porque generan una amplia expectativa en la designación de candidatos, campañas electorales, entre otros.

En el ámbito local, en cambio, los grupos de poder limitan la competencia. La verdadera contienda se da al interior de éstos sin que las elecciones, en sí mismas, representen ejercicios reñidos por ganar puestos públicos1. Pero no sólo la concentración del poder en manos de unos grupos dificulta la competencia; también los factores sociales tienen su cuota. La marginación, pobreza, migración, población indígena y analfabetismo de amplios sectores de la población; aunado 
con una accidentada geografía del estado principalmente en las regiones altas, dificultan la participación electoral a nivel local2.

* Licenciado en Ciencia Política por la Universidad Autónoma Metropolitana, Maestro en Estudios Sociales (UAM-I) y Maestro en Estudios de Población (UAEH). Becario CONACyT y doctorante en Estudios Sociales por la UAM-I. Catedrático de la Escuela de Ciencias Humanas de la Universidad La Salle Pachuca. Líneas de investigación: procesos electorales y sistemas políticos. Correo electrónico: uam_lore04@hotmail.com 0

Sin embargo, éstas características están presentes en el estado desde hace años y no parecen incidir en todas las elecciones por igual. Por ejemplo, las elecciones municipales son las más participativas y regularmente generan amplias expectativas y competencia que, a la postre, posibilitan procesos de alternancia a nivel micro. No obstante, las votaciones de diputados locales tienen su propia lógica. En ellas, la mayoría de los electores (como se verá más adelante) prefieren abstenerse.

¿Qué factores pueden explicar esta conducta? Una primera reflexión sería que el desempeño laboral de los diputados en su conjunto es mal evaluado por los ciudadanos. No es ningún secreto que para amplios sectores de la población los diputados sean representantes bien remunerados que obedecen a los intereses de sus respectivas cúpulas partidistas antes que a los intereses de sus representados3.

Por ello, es especialmente relevante analizar con detalle el desarrollo de los procesos electorales en Hidalgo. Dentro de este recuento se podrá observar que en el estado es frecuente que los distritos electorales enclavados en la huasteca hidalguense zona eminentemente indígena, rural, marginada y con altos índices de analfabetismo sean los más participativos; conducta que contradice las teorías del comportamiento electoral4.

\section{Elecciones de diputados locales 1993}

En 1993, el estado de Hidalgo se dividía en 15 distritos electorales. De ellos, sólo tres tuvieron una participación mayor de 50\% (distritos VI, XII y XIV en Zacualtipán, Molango y Jacala respectivamente) Llama la atención que los distritos más participativos se encuentren enclavados en la huasteca, lugar caracterizado por su elevada población indígena, marginación y analfabetismo (ver mapas en el apéndice). 
CUADRO I

ELECCIÓN DE DIPUTADOS LOCALES DE MAYORÍA RELATIVA

HIDALGO 1993

\begin{tabular}{|l|c|c|c|c|c|}
\hline DISTRITO & $\begin{array}{c}\text { LISTA } \\
\text { NOMINAL }\end{array}$ & $\begin{array}{c}\text { PARTICIPACION } \\
\text { CIUDADANA }\end{array}$ & $\%$ & ABSTENCION & $\%$ \\
\hline I & 99,306 & 46,840 & 47.17 & 52,446 & 52.83 \\
\hline II & 80,395 & 30,542 & 37.99 & 49,853 & 62.01 \\
\hline III & 119,439 & 38,042 & 31.85 & 81,397 & 68.15 \\
\hline IV & 39,917 & 16,619 & 41.63 & 23,298 & 58.37 \\
\hline V & 98,925 & 33,692 & 34.06 & 65,233 & 65.94 \\
\hline VI & $\mathbf{2 9 , 3 6 0}$ & $\mathbf{1 5 , 0 5 3}$ & $\mathbf{5 1 . 2 7}$ & 14,307 & $\mathbf{4 8 . 7 3}$ \\
\hline VII & 19,413 & 6,500 & 33.48 & 12,913 & 66.52 \\
\hline VIII & 16,359 & 7,069 & 43.21 & 9,290 & 56.79 \\
\hline IX & 45,646 & 20,138 & 44.12 & 25,508 & 55.88 \\
\hline X & 90,946 & 37,934 & 41.71 & 53,012 & 58.29 \\
\hline XI & 86,710 & 29,393 & 33.90 & 57,317 & 66.10 \\
\hline XII & $\mathbf{4 5 , 8 7 5}$ & $\mathbf{2 5 , 4 3 2}$ & $\mathbf{5 5 . 4 4}$ & $\mathbf{2 0 , 4 4 3}$ & $\mathbf{4 4 . 5 6}$ \\
\hline XIII & 49,408 & 20,274 & 41.03 & 29,134 & 58.97 \\
\hline XIV & $\mathbf{3 1 , 7 1 9}$ & $\mathbf{1 7 , 3 3 8}$ & $\mathbf{5 4 . 6 6}$ & $\mathbf{1 4 , 3 8 1}$ & $\mathbf{4 5 . 3 4}$ \\
\hline XV & 25,008 & 12,107 & 48.41 & 12,901 & 51.59 \\
\hline
\end{tabular}

Fuente: elaboración propia con datos del CEDE

En cambio, los distritos III, V y II en Tula, Zimapán y Tulancingo respectivamente, concentraron el mayor porcentaje de abstencionismo en ese año. Esto indica que en distritos con alejados de la huasteca se vota más mientras que en los distritos urbanos se vota menos. Esta hipótesis estará presente en casi todas las elecciones de diputados locales.

En la siguiente gráfica se puede observar una disparidad muy significativa entre el nivel de participación y abstención electoral de los distritos II y $V$, en Tulancingo y Zimapán respectivamente. Ahí, 3 de cada 5 ciudadanos optaron por no sufragar. 


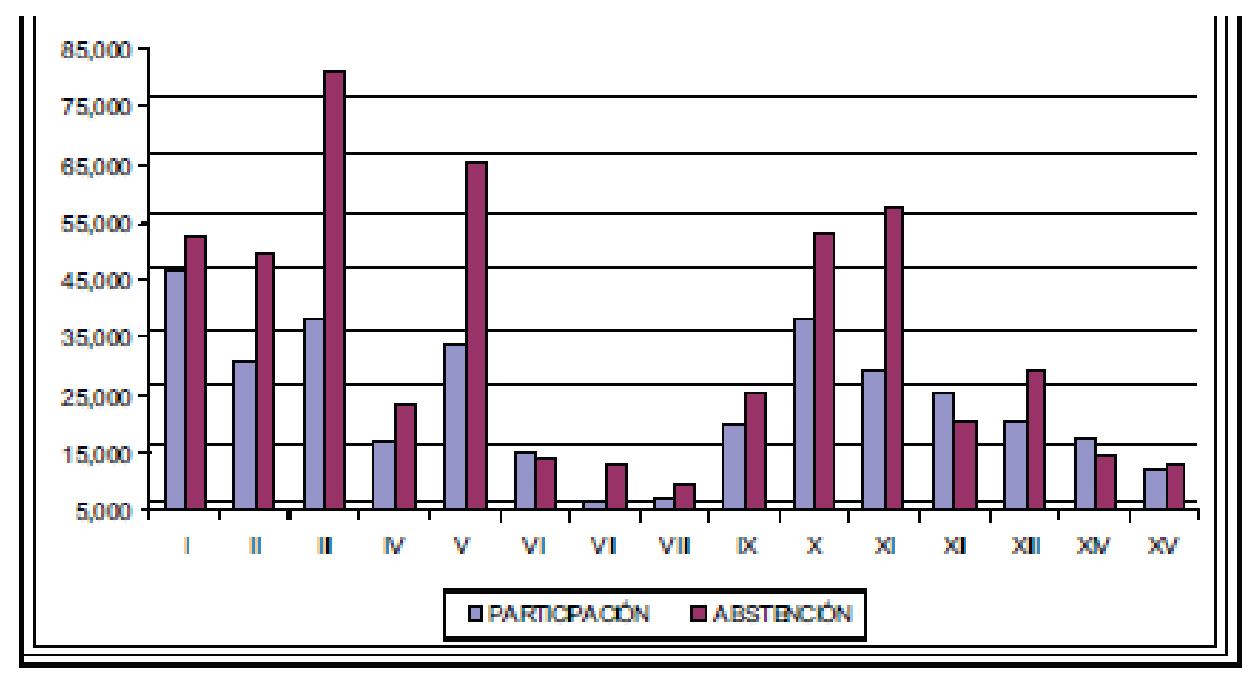

Fuente: elaboración propia con datos del cuadro anterior

En suma, la elección de 1993 tuvo un saldo negativo en cuanto a la participación ciudadana. En la gráfica anterior se muestra una tendencia general hacia el abstencionismo. Hay que recordar que en ese año los procesos electorales eran regulados por la Comisión Federal Electoral Delegación Hidalgo. Ylos resultados electorales, por tanto, no contaban con la validez de una institución autónoma5.

\section{Elecciones de diputados locales 1996}

Para 1996 la situación no se modificó. En el siguiente cuadro se puede observar que, nuevamente, sólo tres distritos superaron el $50 \%$ de participación electoral. Ellos son el IX, XV y XVII en San Agustín Metzquititlán, Molango y Jacala respectivamente. 
CUADRO 2

ELECCIÓN DE DIPUTADOS LOCALES DE MAYORÍA RELATIVA

HIDALGO 1996

\begin{tabular}{|c|c|c|c|c|c|}
\hline DISTRITO & $\begin{array}{c}\text { LISTA } \\
\text { NOMINAL }\end{array}$ & $\begin{array}{l}\text { PARTICIPACION } \\
\text { CIUDADANA }\end{array}$ & $\%$ & ABSTENCION & $\%$ \\
\hline 1 & 81,395 & 32,040 & 39.36 & 49,355 & 60.64 \\
\hline II & 72,215 & 28,894 & 40.01 & 43,321 & 59.99 \\
\hline III & 94,846 & 32,047 & 33.79 & 62,799 & 66.21 \\
\hline IV & 72,685 & 23,147 & 31.85 & 49,538 & 68.15 \\
\hline $\mathrm{V}$ & 73,038 & 23,115 & 31.65 & 49,923 & 68.35 \\
\hline $\mathrm{VI}$ & 46,715 & 20,606 & 44.11 & 26,109 & 55.89 \\
\hline VII & 33,113 & 14,907 & 45.02 & 18,206 & 54.98 \\
\hline VIII & 20,195 & 6,825 & 33.80 & 13,370 & 66.20 \\
\hline IX & 23,008 & 12,118 & 52.67 & 10,890 & 47.33 \\
\hline $\bar{x}$ & 48,366 & 19,410 & 40.13 & 28,956 & 59.87 \\
\hline$X \mid$ & 66,405 & 25,454 & 38.33 & 40,951 & 61.67 \\
\hline $\mathrm{XII}$ & 48,138 & 14,121 & 29.33 & 34,017 & 70.67 \\
\hline XIII & 96,001 & 43,960 & 45.79 & 52,041 & 54.21 \\
\hline$\overline{\mathrm{XN}}$ & 90,815 & 25,282 & 27.84 & 65,533 & 72.16 \\
\hline$\overline{X V}$ & 44,207 & 24,968 & 56.48 & 19,239 & 43.52 \\
\hline$\overline{\mathrm{XVI}}$ & 57,710 & 21,842 & 37.85 & 35,868 & 62.15 \\
\hline $\mathrm{XVI}$ & 28,117 & 15,641 & 55.63 & 12,476 & 44.37 \\
\hline XVIII & 35,953 & 16,485 & 45.85 & 19,468 & 54.15 \\
\hline
\end{tabular}

Fuente: elaboración propia con datos del IEE

Es evidente que los distritos correspondientes a la huasteca (Molango y Jacala) son los más participativos en elecciones. ¿Qué ocurre en ese lugar? y ¿cómo explicar ese comportamiento electoral? Como se ha visto en 1993 y 1996 esos distritos son muy proclives a participar en elecciones. Quizá la presencia de los partidos sea muy fuerte $y$, por tanto, estimulan una competencia en donde los ciudadanos se involucran con cierto frenesí. Pero también existe otra lectura. Puede ser que los mecanismos de manipulación y alteración de resultados se encuentren presentes justamente ahí. Alejados de las ciudades, en donde es muy complicado comprobar un fraude electoral. 
GRAFICA 2

PAFTICIPACIÓN Y ABSTENCIÓN (absolutos)

ELECCIÓN DE DIPUTADOS LOCALES, 1996

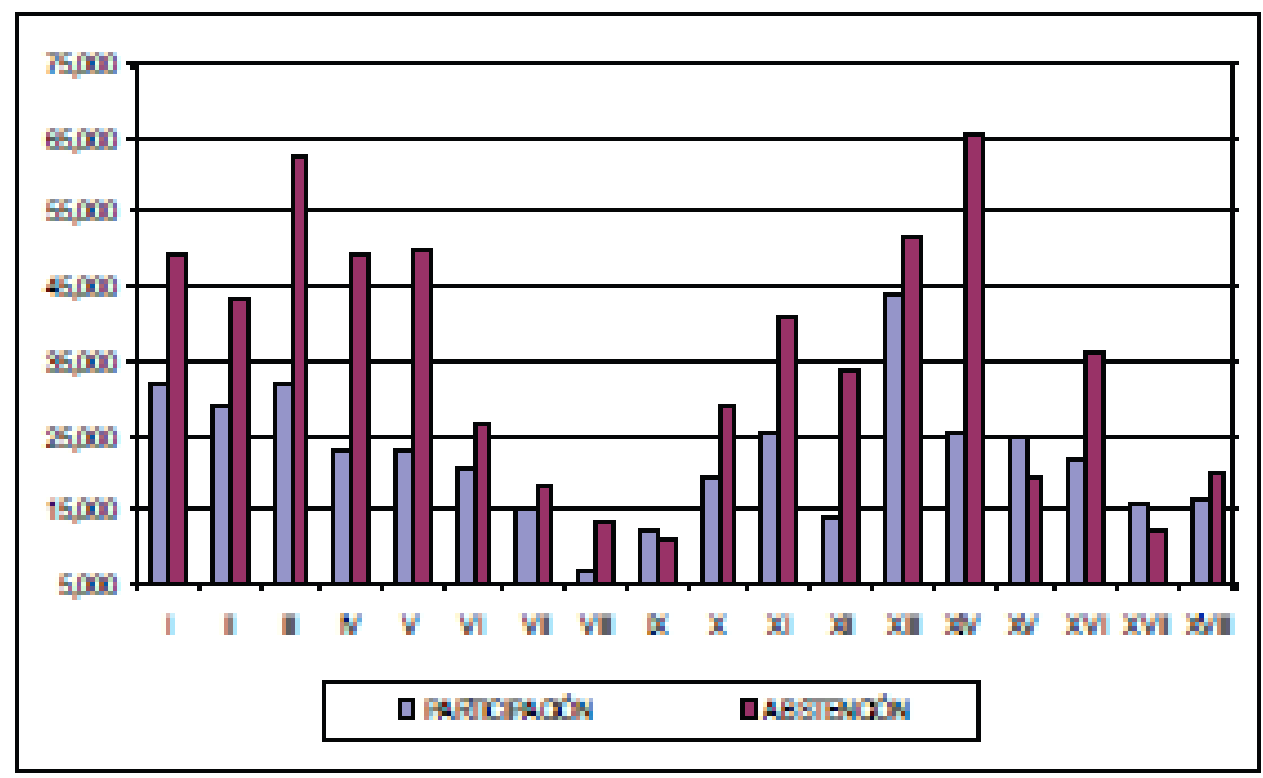

Fuente: elaboracion propia con datos del cuadro anterior

La gráfica anterior es muy similar a la de 1993. A pesar de la modificación que realizó la autoridad electoral (IEE) en 1995, implementando una nueva distritación electoral, los niveles de participación se mantuvieron bajos.

\section{Elecciones de diputados locales 1999}

Para 1999 los niveles de participación se incrementaron significativamente. Esta conducta contraria a la de los procesos electorales anteriores puede explicarse, en parte, por el ambiente previo a la elección federal del año 2000. Especialmente cuando las campañas federales tuvieron una larga duración en los medios masivos de comunicación. En 1999, las tendencias se modificaron radicalmente. Nueve distritos electorales rebasaron el $50 \%$ de participación electoral, sin embargo, el resto tuvieron niveles muy cercanos a esa proporción. 
CUADRO 3

ELECCIÓN DE DIPUTADOS LOCALES DE MAYORÍA RELATIVA

HIDALGO 1999

\begin{tabular}{|c|c|c|c|c|c|}
\hline DISTRITO & $\begin{array}{c}\text { LISTA } \\
\text { NOMINAL }\end{array}$ & $\begin{array}{l}\text { PARTICIPACION } \\
\text { CIUDADANA }\end{array}$ & $\%$ & ABSTENCION & $\%$ \\
\hline 1 & 98,816 & 51,363 & 51.98 & 47,453 & 48.02 \\
\hline II & 91,611 & 49,433 & 53.96 & 42,178 & 46.04 \\
\hline III & 115,337 & 59,820 & 51.87 & 55,517 & 48.13 \\
\hline IV & 85,973 & 42,432 & 49.36 & 43,541 & 50.64 \\
\hline V & 87,274 & 42,178 & 48.33 & 45,096 & 51.67 \\
\hline VI & 54,991 & 28,486 & 51.80 & 26,505 & 48.20 \\
\hline VII & 39,536 & 21,381 & 54.08 & 18,155 & 45.92 \\
\hline VIII & 25,026 & 12,212 & 48.80 & 12,814 & 51.20 \\
\hline $\mathrm{IX}$ & 26,767 & 14,846 & 55.46 & 11,921 & 44.54 \\
\hline $\bar{x}$ & 56,981 & 27,226 & 47.78 & 29,755 & 52.22 \\
\hline $\mathrm{XI}$ & 76,691 & 36,425 & 47.50 & 40,266 & 52.50 \\
\hline XII & 60,346 & 27,053 & 44.83 & 33,293 & 55.17 \\
\hline $\mathbf{X I I I}$ & 115,196 & 70,675 & 61.35 & 44,521 & 38.65 \\
\hline XIV & 108,523 & 49,446 & 45.56 & 59,077 & 54.44 \\
\hline $\mathrm{XV}$ & 52,105 & 32,125 & 61.65 & 19,980 & 38.35 \\
\hline $\mathrm{XVI}$ & 71,120 & 34,085 & 47.93 & 37,035 & 52.07 \\
\hline XVI & 33,008 & 18,480 & 55.99 & 14,528 & 44.01 \\
\hline XVIII & 43,736 & 21,026 & 48.07 & 22,710 & 51.93 \\
\hline
\end{tabular}

Fuente: elaboración propia con datos del IEE

Esta elección tuvo una participación destacada de la ciudadanía hasta entonces renuente con la elección de diputados locales. En ciudades como Pachuca y Tulancingo los porcentajes de participación aumentaron considerablemente (distritos I, II y III respectivamente). Molango y Huejutla no fueron la excepción. En esos distritos (XIII y XV) que agrupan a media docena de municipios de la huasteca, más del $60 \%$ de ciudadanos sufragaron manteniendo constantes los altos niveles de respaldo electoral.

Sólo el distrito XII (Tizayuca) rebasó el 55\% de abstencionismo pero en términos generales, la elección de 1999 fue más participativa que las de 1993 y 1996. Como puede verse en la siguiente gráfica las líneas de participación y abstención se encuentran casi en el mismo nivel. 


\section{GRÁFICA 3 \\ PARTICIPACIÓN Y ABSTENCIÓN (absolutos) \\ ELECCIÓN DE DIPUTADOS LOCALES, 1999}

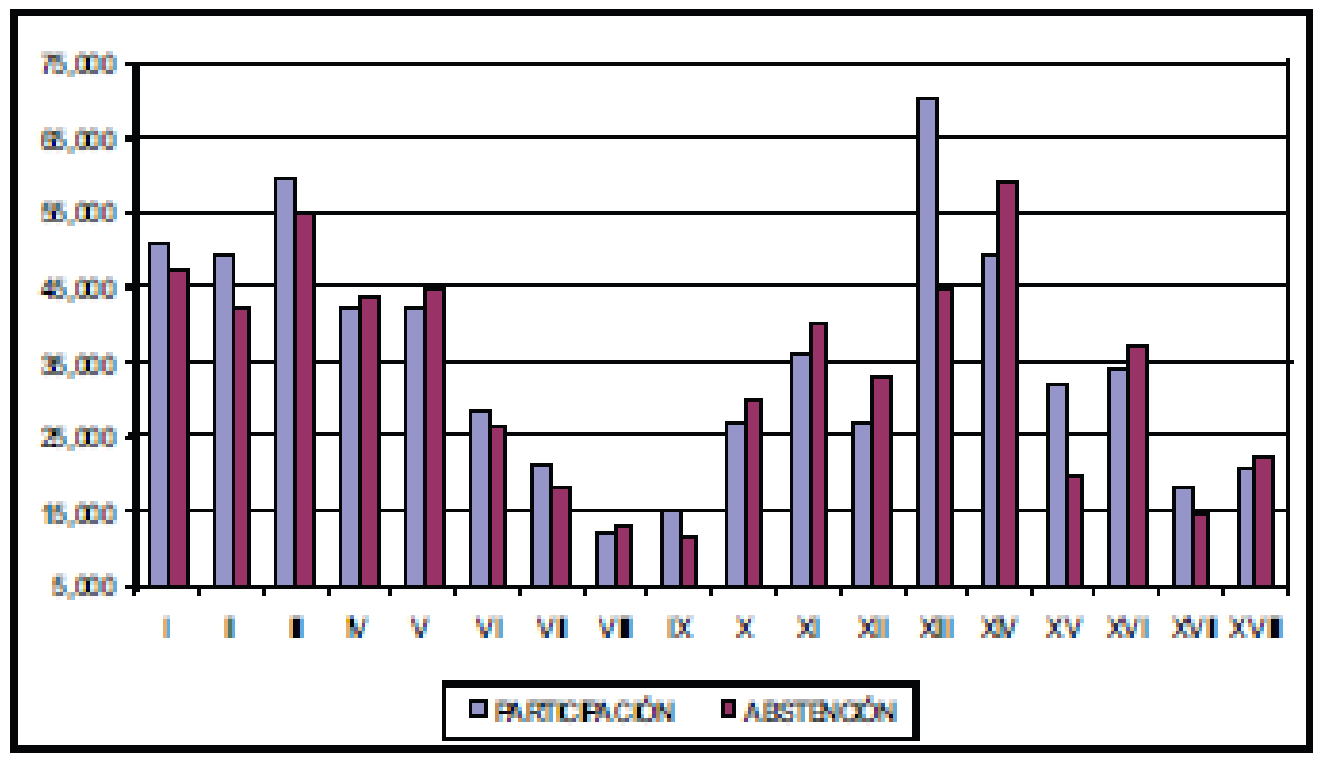

Fuente: elaboración propia con datos del cuadro anterior

La gráfica 3, muestra claramente un comportamiento distinto en comparación con las elecciones de 1993 y 1996. Es claro que la diferencia entre participación y abstención se cruza de manera regular sin tanta diferencia.

\section{Elecciones de diputados locales 2002}

Para el año 2002 el abstencionismo volvió a ganar terreno. Nuevamente tres distritos rebasaron el $50 \%$ de participación electoral (San Agustín Metzquititlán, Molango y Jacala). El resto no sólo fue ampliamente abstencionista sino que superó el nivel hasta entonces conocido -en el estado de Hidalgo- de apatía electoral. En once distritos electorales más del $60 \%$ de los ciudadanos no votaron. El distrito con más ausentismo fue el XIV con cabecera en Actopan, con $74 \%$. 
CUADRO 4

ELECCIÓN DE DIPUTADOS LOCALES DE MAYORÍA RELATIVA HIDALGO 2002

\begin{tabular}{|c|c|c|c|c|c|}
\hline DISTRTO & $\begin{array}{c}\text { LISTA } \\
\text { NOMINAL }\end{array}$ & $\begin{array}{l}\text { PARTICIPACION } \\
\text { CIUDADANA }\end{array}$ & $\%$ & ABSTENCION & $\%$ \\
\hline 1 & 108,283 & 36,945 & 34.12 & 71,338 & 65.88 \\
\hline TII & 105,728 & 33,224 & 31.42 & 72,504 & 68.58 \\
\hline III & 130,994 & 37,779 & 28.84 & 93,215 & 71.16 \\
\hline $\mathrm{N}$ & 95,887 & 27,585 & 28.77 & 68,302 & 71.23 \\
\hline $\mathrm{V}$ & 97,581 & 27,175 & 27.85 & 70,406 & 72.15 \\
\hline $\mathrm{Vl}$ & 59,718 & 23,488 & 39.33 & 36,230 & 60.67 \\
\hline VII & 43,345 & 19,778 & 45.63 & 23,567 & 54.37 \\
\hline VIII & 29,110 & 10,856 & 37.29 & 18,254 & 62.71 \\
\hline$\overline{I x}$ & 29,436 & 14,743 & 50.08 & 14,693 & 49.92 \\
\hline $\bar{x}$ & 62.233 & 25,525 & 41.02 & 36,708 & 58.98 \\
\hline $\mathrm{X} \mid$ & 82,632 & 24,437 & 29.57 & 58,195 & 70.43 \\
\hline XII & 68,859 & 19,263 & 27.97 & 49,596 & 72.03 \\
\hline XIII & 128,032 & 58,779 & 45.91 & 69,253 & 54.09 \\
\hline$\overline{X N}$ & 121,433 & 30,671 & 25.26 & 90,762 & 74.74 \\
\hline $\mathrm{XV}$ & 56,325 & 30,283 & 53.76 & 26,042 & 46.24 \\
\hline$\overline{X V \mid}$ & 80,511 & 33,722 & 41.88 & 46,789 & 58.12 \\
\hline XVI & 36,064 & 19,744 & 54.75 & 16,320 & 45.25 \\
\hline XVIII & 48,071 & 18,406 & 38.29 & 29,665 & 61.71 \\
\hline
\end{tabular}

Fuente: elaboración propia con datos del IEE

Llama la atención que seis distritos presenten niveles mayores a $70 \%$ de apatía electoral. Principalmente en distritos urbanos (Tulancingo, Tula, Tepeji del Río, Apan, Tizayuca y Actopan). Lo que pondría en entredicho la hipótesis que se sostenía anteriormente. Con los datos de la elección local de 2002 se puede deducir que en los distritos más urbanos se vota menos que en los distritos rurales. De este modo, se observa que en un periodo de nueve años el comportamiento electoral cambió radicalmente. Ahora el abstencionismo es un fenómeno en crecimiento en regiones urbanas y rurales por igual 7 .

La gráfica 4 muestra una diferencia considerable entre los indicadores estudiados. La línea de la abstención en la mayoría de los distritos supera a la línea participativa. El distrito III (Tulancingo) y XIV (Actopan) son los que más resaltan por su abstención. 


\section{GRÁFICA 4 \\ PARTICIPACIÓN Y ABSTENCIÓN (absolutos) \\ ELECCIÓN DE DIPUTADOS LOCALES, 2002}

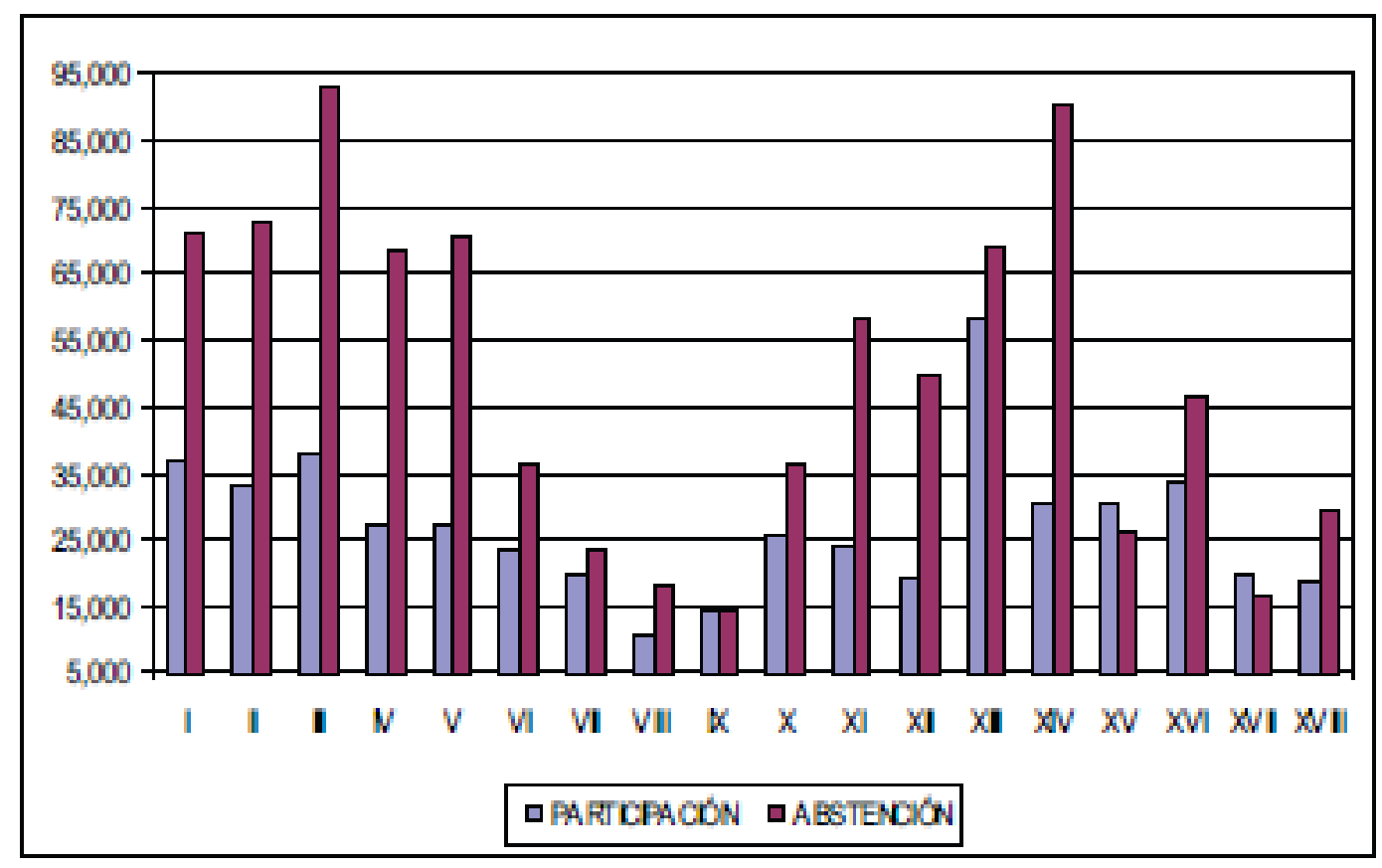

El elevado abstencionismo en 2002 en casi todo el territorio estatal se presentó incluso con mayor intensidad en la elección federal de 2003. Estas dos elecciones (2002 local y 2003 federal) han sido las menos respaldadas por el electorado en el estado de Hidalgo8.

Ese desencanto del electorado llamó la atención de numerosos especialistas en materia electoral. Quienes adjudicaron, en general, un sentimiento de desilusión con todos los partidos políticos como causa principal del elevado ausentismo en las urnas9. En otras palabras, las expectativas generadas por la alternancia en el nivel federal no vinieron emparejadas - al menos, no en Hidalgo - de una mayor participación en los procesos electorales. La vía electoral que hizo posible el fin del régimen dominante del PRI no pudo ser refrendada en las siguientes elecciones de 2002 y 2003.

\section{Elecciones de diputados locales 2005}

En la elección de 2005 los niveles de participación aumentaron. Nuevamente el proceso federal de 2006 incidió positivamente en el interés por la participación electoral. En 2005, sólo 5 distritos rebasaron el $60 \%$ de abstención. Los restantes oscilaron entre el 40 y $50 \%$ como lo muestra el siguiente cuadro. 
CUADRO 5

ELECCIÓN DE DIPUTADOS LOCALES DE MAYORÍA RELATIVA HIDALGO 2005

\begin{tabular}{|c|c|c|c|c|c|}
\hline DIST RITO & $\begin{array}{c}\text { LISTA } \\
\text { NOMINAL } \\
\end{array}$ & $\begin{array}{l}\text { PARTICIPACION } \\
\text { CIUDADANA } \\
\end{array}$ & $\%$ & ABSTENCION & $\%$ \\
\hline I & 123,333 & 57,379 & 46.52 & 64,964 & 53.48 \\
\hline II & 128,696 & $58,8.43$ & 45.72 & 69,852 & 54.28 \\
\hline III & 147,359 & 52379 & 35.55 & 94,980 & 64.45 \\
\hline IV & 106,445 & 46,842 & 44.01 & 59,603 & 55.99 \\
\hline $\bar{v}$ & 108,009 & 42,116 & 38.99 & 65,893 & 61.01 \\
\hline VI & 64,830 & 28,278 & 43.62 & 36,552 & 56.38 \\
\hline VII & 47,719 & 21,458 & 44.97 & 26,261 & 55.03 \\
\hline VIIII & 31,786 & 14,908 & 46.90 & 16,878 & 53.10 \\
\hline$\overline{I X}$ & 31,677 & 16,437 & 51.89 & 15,240 & 48.11 \\
\hline $\bar{x}$ & 69,724 & 31,937 & 45.80 & 37,787 & 54.20 \\
\hline$\overline{X I}$ & 88,327 & 35,850 & $\$ 0.59$ & 52,477 & 59.41 \\
\hline$\overline{X \|}$ & 76,261 & 29,280 & 38.39 & 46,981 & 61.61 \\
\hline XIII & 141,283 & 82,687 & 58.53 & 58,596 & 41.47 \\
\hline $\mathrm{XIV}$ & 134,697 & 59,448 & 44.13 & 75,249 & 55.87 \\
\hline $\mathrm{XV}$ & 60,917 & 37,195 & 61.06 & 23,722 & 38.94 \\
\hline$\overline{X V \mid}$ & 89,844 & 35,602 & 39.63 & 54,242 & 60.37 \\
\hline XVII & 39,168 & 22,794 & 58.20 & 16,374 & 41.80 \\
\hline XVIII & 54,037 & 20,750 & 38.40 & 33,287 & 61.60 \\
\hline
\end{tabular}

Fuente: elaboración propia con datos del IEE

Con esta elección se ponen de manifiesto algunas tendencias, por ejemplo, el distrito XIII y XIV (correspondientes a Huejutla y Molango) en la huasteca hidalguense aparecen - una vez más - como los más participativos. Es muy peculiar que en esa zona, marcada por limitaciones sociales, geográficas y demográficas la participación electoral sea tan elevada. 
GRÁFICA 5

PARTICIPACIÓN Y ABSTENCIÓN (absolutos)

ELECCIÓN DE DIPUTADOS LOCALES, 2005

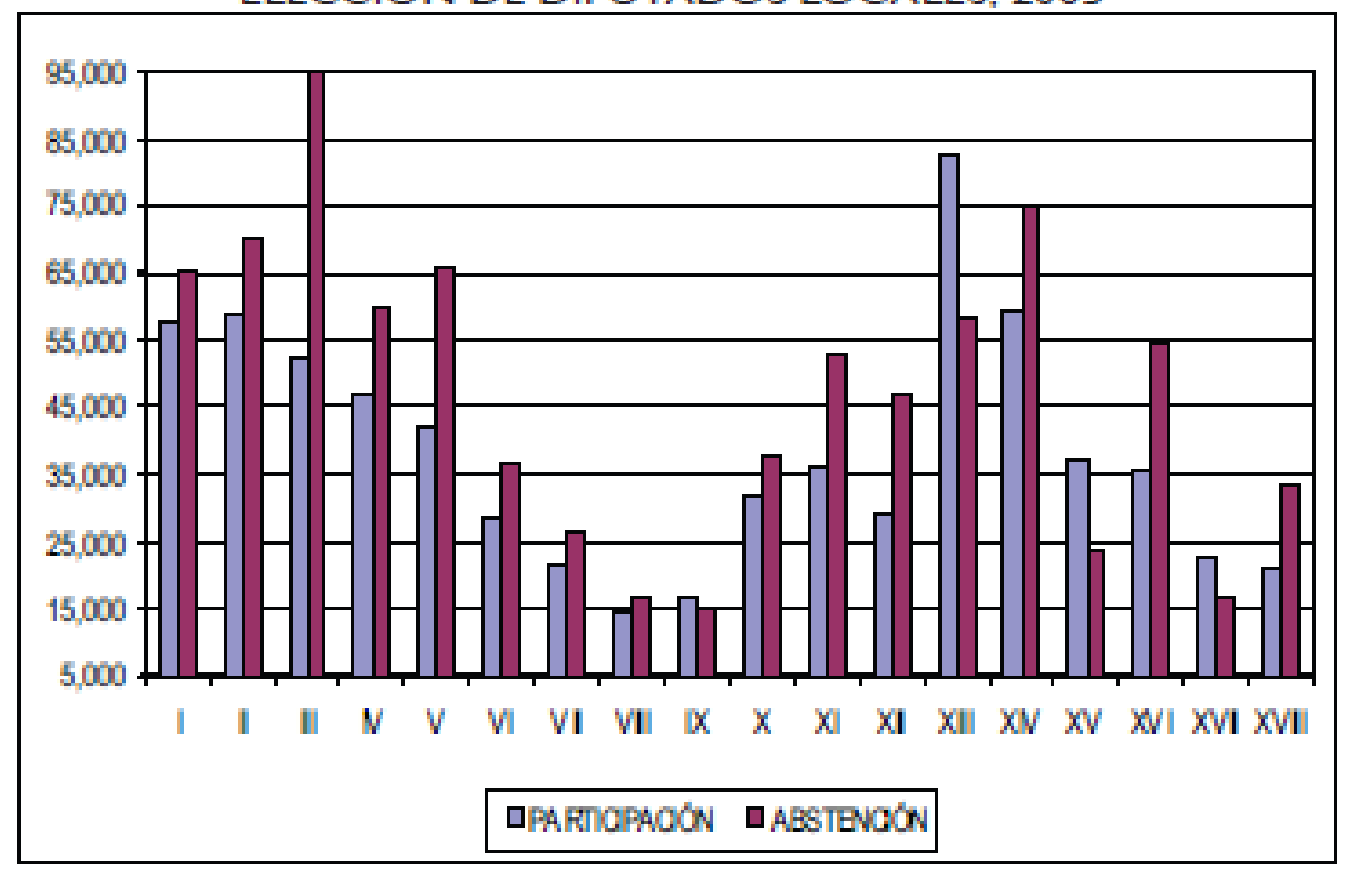

Fuente: elaboración propia con datos del cuadro anterior

En cambio los distritos III y XV correspondientes a ciudades grandes como Tulancingo y Actopan aparecen como los más abstencionistas. Gracias al recuento anterior de cuatro elecciones de diputados locales (1993, 1996,2002 y 2005) se puede observar una dinámica muy específica en cada elección. Los patrones de comportamiento electoral fueron muy similares en 1993, 1996 y 2002, mientras que en 1999 y 2005, cambiaron radicalmente. En el primer periodo los distritos más participativos fueron los pertenecientes a la huasteca hidalguense mientras que en los restantes distritos el abstencionismo era un fenómeno en crecimiento. No obstante, en 1999 y 2005 los procesos electorales federales parecen incidir de manera positiva en los índices de participación electoral estimulando a los electores a participar.

Uno de los principales hallazgos de este recuento es que en la huasteca la participación electoral es muy elevada. Una posible lectura, aunque aventurada y sin los suficientes elementos para sostenerla, sugeriría que en los distritos alejados es más factible alterar los datos, manipular los resultados y simular una participación electoral copiosa. 
Ese posible escenario, sin embargo, no es suficiente para explicar un fenómeno tan complejo como lo es el abstencionismo. Habría que tomar en cuenta otros elementos como la fortaleza de los partidos políticos a nivel distrital, la designación de los candidatos, la influencia de los grupos de poder local, entre otros.

Siguiendo la anterior tendencia, es muy probable que en las próximas elecciones de diputados locales en 2008 los niveles de abstención rebasen el 60\% como ocurrió en los procesos locales que no coincidieron con campañas de elecciones federales. Aunado a ello, habría que observar el comportamiento electoral de los distritos correspondientes a la huasteca hidalguense ya que resulta difícil de entender cómo una región con esas características se distinga por ser muy participativa en elecciones.

\section{Consideraciones finales}

1. Existe una significativa variación del abstencionismo entre una elección y otra. Los distri-

tos que pueden ser catalogados como los más participativos en una elección aparecen en

el siguiente proceso como los más abstencionistas.

2. En elecciones locales ocurre algo peculiar; los distritos enclavados en la huasteca hidal-

guense, zona eminentemente indígena, rural, marginada y analfabeta son los más participa-

tivos. Habría que ponderar con detalle como se relacionan estos dos fenómenos.

3. Con este primer acercamiento a los datos electorales en un periodo de 1993 a 2005 se

puede asegurar que el abstencionismo se relaciona con otros factores hasta ahora no

abordados como: la influencia de los grupos políticos locales, la fortaleza de los partidos

políticos en los distritos y los procesos de designación de candidatos, entre otros.

\section{Apéndice}

Mapa 1 Regiones del Estado de Hidalgo 
Mapa 2 Distritos Electorales Locales

Cuadro Lista de municipios que integran los Distritos Electorales Locales

Mapa 3 Índice de marginación municipal 2005

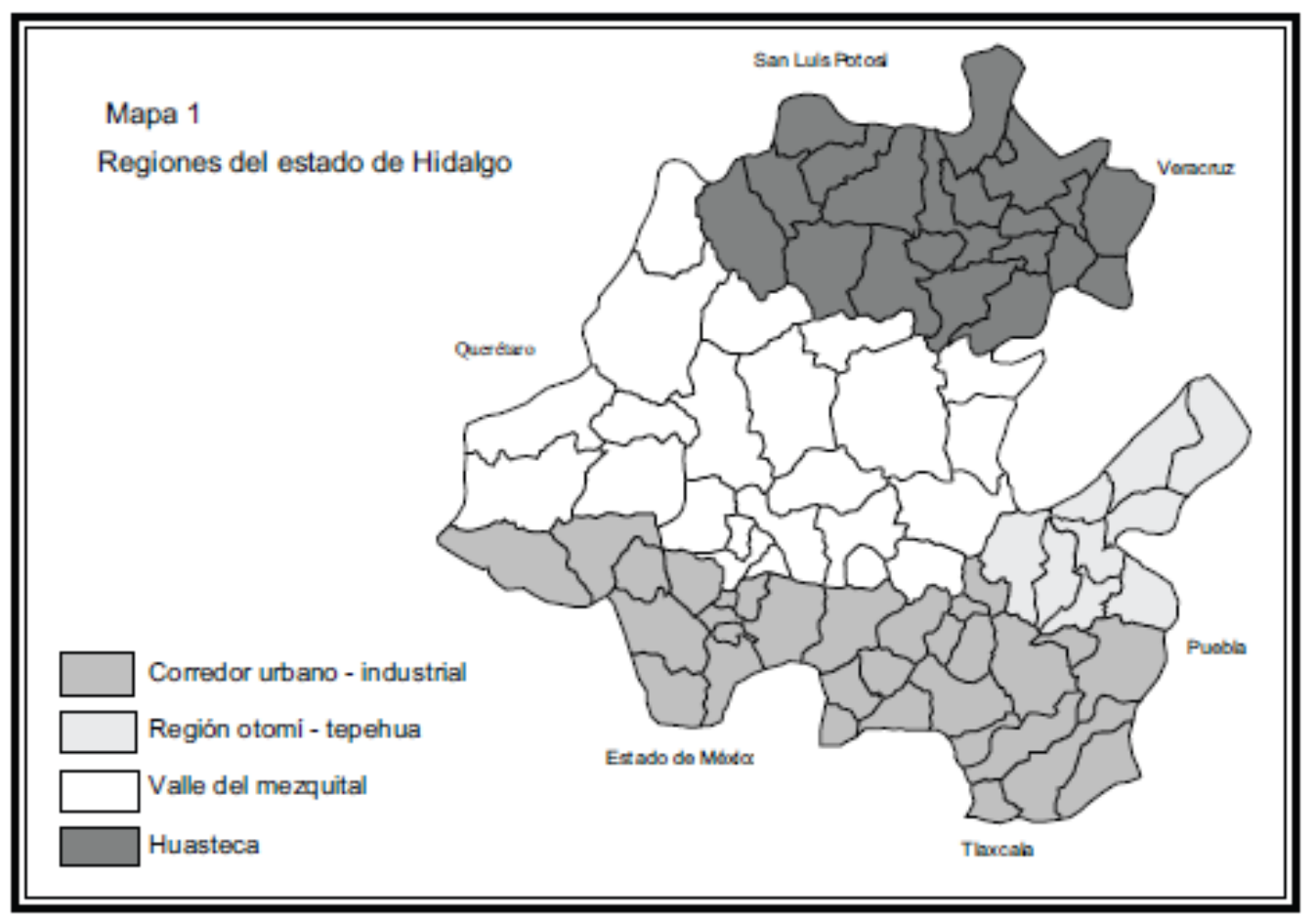




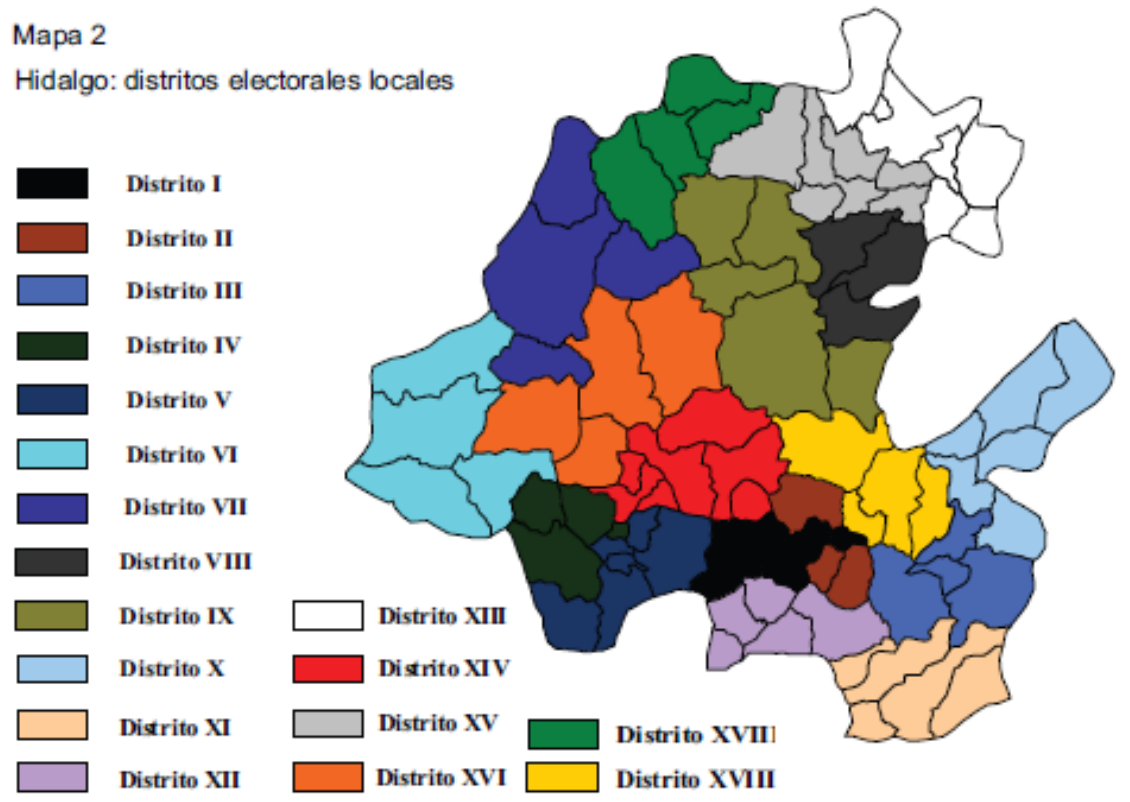




\begin{tabular}{|l|l|l|}
\hline \multicolumn{1}{|c|}{ I DISTRITO } & \multicolumn{1}{c|}{ II DISTRITO } & \multicolumn{1}{c|}{ III DISTRITO } \\
\hline PACHUCA PONIENTE & PACHUCA ORIENTE & TULANCINGO DE BRAVO \\
\hline MINERAL DEL MONTE & MINERAL DE LA REFORMA & $\begin{array}{l}\text { CUAUTEPEC DE } \\
\text { HINOJOSA }\end{array}$ \\
\hline SAN AGUSTIN TLAXIACA & EPAZOYUCAN & SANTIAGO TULANTEPEC \\
\hline & & SINGUILICAN \\
\hline
\end{tabular}

\begin{tabular}{|l|l|l|}
\hline \multicolumn{1}{|c|}{ IV DISTRITO } & \multicolumn{1}{c|}{ V DISTRITO } & \multicolumn{1}{c|}{ VI DISTRITO } \\
\hline TULA DE ALLENDE & $\begin{array}{l}\text { TEPEJI DEL RIO DE } \\
\text { OCAMPO }\end{array}$ & HUICHAPAN \\
\hline TLAHUELILPAN & TETEPANGO & TECOZAUTLA \\
\hline TEPETITLAN & ATOTONILCO DE TULA & NOPALA DE VILLAGRAN \\
\hline TEZONTEPEC DE ALDAMA & ATITALAQUIA & CHAPANTONGO \\
\hline & AJACUBA & \\
\hline & TLAXCOAPAN & \\
\hline
\end{tabular}

\begin{tabular}{|l|l|l|}
\hline \multicolumn{1}{|c|}{ VII DISTRITO } & \multicolumn{1}{c|}{ VIII DISTRITO } & \multicolumn{1}{c|}{ IX DISTRITO } \\
\hline ZIMAPAN & $\begin{array}{l}\text { ZACUALTIPAN DE } \\
\text { ANGELES }\end{array}$ & $\begin{array}{l}\text { SAN AGUSTIN } \\
\text { METZQUITITLAN }\end{array}$ \\
\hline TASQUILLO & TIANGUISTENGO & METZTITLAN \\
\hline NICOLAS FLORES & XOCHICOATLAN & ELOXOCHITLAN \\
\hline PACULA & & JUAREZ HIDALGO \\
\hline & & TLAHUILTEPA \\
\hline
\end{tabular}

\begin{tabular}{|l|l|l|}
\hline \multicolumn{1}{|c|}{ X DISTRITO } & \multicolumn{1}{c|}{ XI DISTRITO } & \multicolumn{1}{c|}{ XIIDISTRITO } \\
\hline TENANGO DE DORIA & APAN & TIZAYUCA \\
\hline ACAXOCHITLAN & EMILIANO ZAPATA & ZAPOTLAN DE JUAREZ \\
\hline METEPEC & ALMOLOYA & TOLCAYUCA \\
\hline HUEHUETLA & TEPEAPULCO & VILLA DE TEZONTEPEC \\
\hline $\begin{array}{l}\text { SAN BARTOLO } \\
\text { TUTOTEPEC }\end{array}$ & TLANALAPA & ZEMPOALA \\
\hline $\begin{array}{l}\text { AGUA BLANCA DE } \\
\text { ITURBIDE }\end{array}$ & & \\
\hline
\end{tabular}

\begin{tabular}{|c|c|c|}
\hline XIII DISTRITO & XIV DISTRITO & XV DISTRITO \\
\hline HUEJUTLA DE REYES & ACTOPAN & MOLANGO DE ESCAMILLA \\
\hline HUAUTLA & SAN SALVADOR & $\begin{array}{l}\text { TEPEHUACAN DE } \\
\text { GUERRERO }\end{array}$ \\
\hline YAHUALICA & EL ARENAL & CALNALI \\
\hline ATLAPEXCO & SANTIAGO DE ANAYA & LOLOTLA \\
\hline XOCHIATIPAN & $\begin{array}{l}\text { MIXQUIAHUALA DE } \\
\text { JUEREZ }\end{array}$ & HUAZALINGO \\
\hline SANFELIPE ORIZATLAN & FRANCISCO I. MADERO & TLANCHINOL \\
\hline JALTOCAN & PROCESO DE OBREGON & \\
\hline XVI DISTRITO & XVII DISTRITO & XVIII DISTRITO \\
\hline IXMIQUILPAN & JACALA DE LEDEZMA & ATOTONILCO EL GRANDE \\
\hline ALFAJAYUCAN & CHAPULHUACAN & HUASCA DE OCAMPO \\
\hline EL CARDONAL & PISAFLORES & OMITLAN DE JUÁREZ \\
\hline CHILCUAUTLA & LA MISIÓN & MINERAL DEL CHICO \\
\hline & & ACATLAN \\
\hline
\end{tabular}




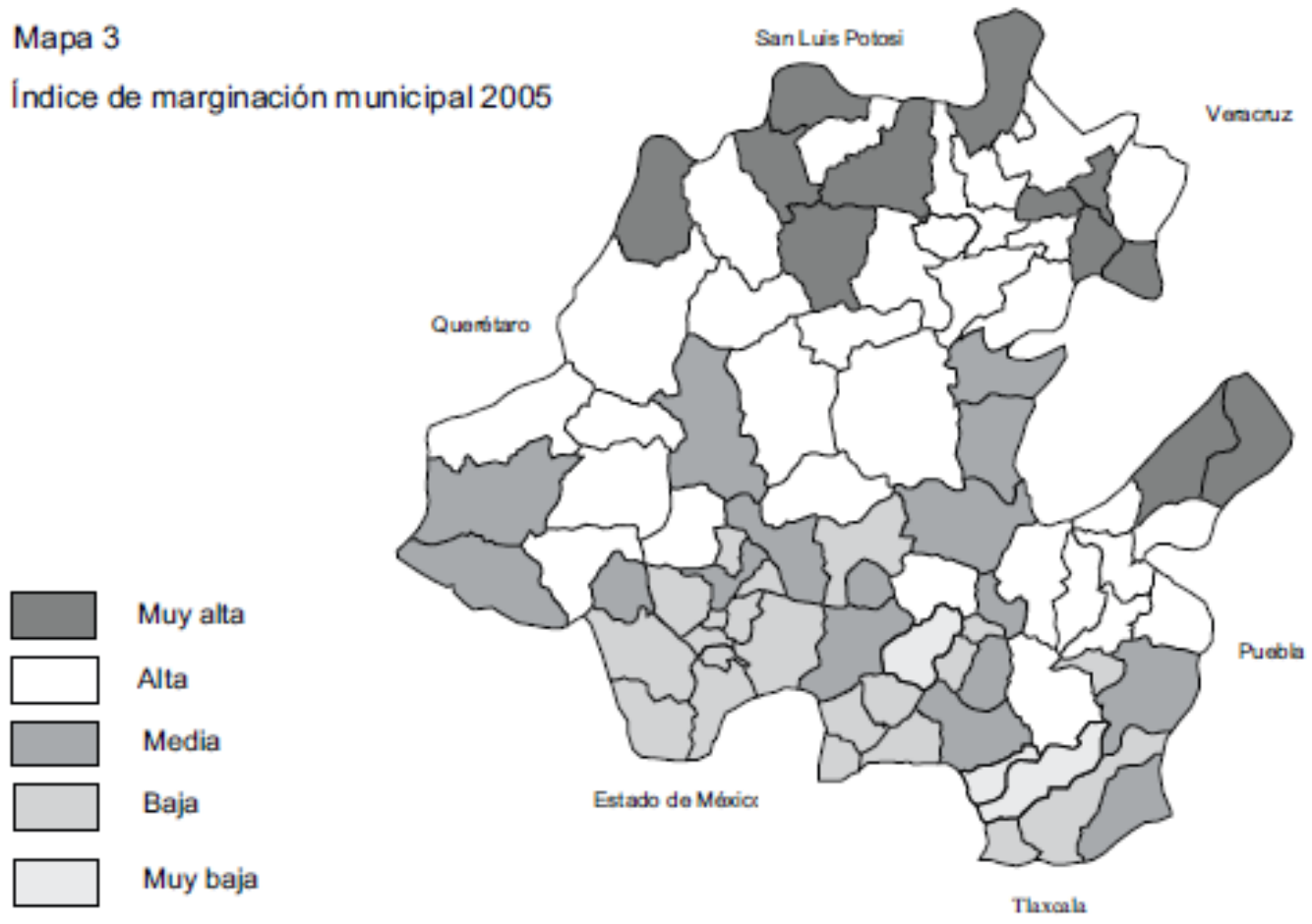

\section{NOTAS}

1 Ver Vargas González, Pablo. Hidalgo las dificultades de la transición política, Ed. Universidad de Guadalajara, Universidad Autónoma del Estado de Hidalgo, México 1998.

2 En un estudio reciente se utilizó el índice de correlación Pearson para identificar el nivel de influencia de la participación electoral en poblaciones con elevada marginación, pobreza, migración y población indígena. Encontrando, en general, una correlación positiva y significativa en algunas regiones del estado. Ver López Rivera, Enrique. ¿Quienes votan y quiénes no votan? la participación electoral de la población marginada, indígena y migrante del estado de Hidalgo, Ponencia presentada en el XIX Congreso Nacional y II Internacional de Estudios Electorales, 20-23 noviembre, Guadalajara Jalisco.

3 Al respecto puede verse la Encuesta Nacional sobre Cultura Política y Prácticas Ciudadanas 2001 elaborada por INEGI y la Secretaría de Gobernación. 
4 Ver Anduiza Eva y Agustí Bosch Comportamiento político y electoral Ed Ariel, Madrid 2004.

5 Una recopilación sobre las instituciones que han organizado las elecciones en el estado puede verse en Alcalá Montaño, Alfredo Hidalgo "Entre el autoritarismo y la democracia. El Instituto Estatal Electoral y la Alternancia Municipal en el estado (1995-2002)" en Revista Mexicana de Estudios Electorales No. 4, Julio - diciembre 2004.

6 El fraude electoral es por naturaleza muy difícil de comprobar. Sin embargo, son múltiples y muy variados los ejemplos de alteración y manipulación de votos en las regiones más alejadas del estado. Un amplio recuento de éstas prácticas puede verse en Vargas González, Pablo. Hidalgo elecciones y reforma política 19792000, Ed. Cámara de Diputados LVIII Legislatura, Universidad Autónoma del Estado de Hidalgo, México 2003.

7 Sobre el tema puede consultarse del autor, El perfil demográfico del abstencionista hidalguense. El caso de la elección federal de 2003. Tesis para obtener el título de Maestro en Estudios de Población por la Universidad Autónoma del Estado de Hidalgo, México 2007.

8 Idem, p. 176.

9 Palma, Esperanza ¿Quiénes apoyan a los partidos en México? Un análisis de las bases sociales de los partidos después de la alternancia, en Polis Volumen 2, número 2, segundo semestre 2006, Depto de Sociología de la Universidad Autónoma Metropolitana, México 2006.

10 Ver Córdova Vianello, Lorenzo (2006) Breves apuntes conceptuales sobre el abstencionismo en México en Serie Breviarios de Cultura Política Democrática, Instituto Estatal Electoral del Estado de México (IEEM) México.

\section{FUENTES DE CONSULTA}

AnduizaEva y Agustí Bosch, (2004), Comportamiento político y electoral. Ed Ariel, Madrid.

AlcaláMontaño, A. (2004), "Hidalgo entre el autoritarismo y la democracia. El Instituto Estatal Electoral y la Alternancia Municipal en el Estado 1995 - 2002" en Revista Mexicana de Estudios Electorales No. 4, Julio - diciembre 2004.

CórdovaVianello, Lorenzo, (2006), Breves apuntes conceptuales sobre el abstencionismo en México en Serie Breviarios de Cultura Política Democrática, Instituto Estatal Electoral del Estado de México (IEEM) México. 
INEGI. (2003), Encuesta Nacional sobre Cultura Política y Prácticas Ciudadanas 2001 México : INEGI y SEGOG.

LópezRivera, Enrique, ¿Quienes votan y quiénes no votan? la participación electoral de la población marginada, indígena y migrante del estado de Hidalgo, Ponencia presentada en el XIX Congreso Nacional y II Internacional de Estudios Electorales, 20-23 noviembre, Guadalajara Jalisco.

-. (2007), El perfil demográfico del abstencionista hidalguense. El caso de la elección federal de 2003. Tesis para obtener el Título de Maestro en Estudios de Población por la Universidad Autónoma del Estado de Hidalgo, México.

Palma, Esperanza (2006), ¿Quiénes apoyan a los partidos en México? Un análisis de las bases sociales de los partidos después de la alternancia, en Polis Volumen 2, número 2, segundo semestre 2006, México: Depto de Sociología de la Universidad Autónoma Metropolitana.

VargasGonzález, Pablo (1998), Hidalgo las dificultades de la transición política, , México: Universidad Autónoma del Estado de Hidalgo

-. (2003), Hidalgo elecciones y reforma política 1979-2000, Ed. Cámara de Diputados LVIII Legislatura, México: Universidad Autónoma del Estado de Hidalgo. 